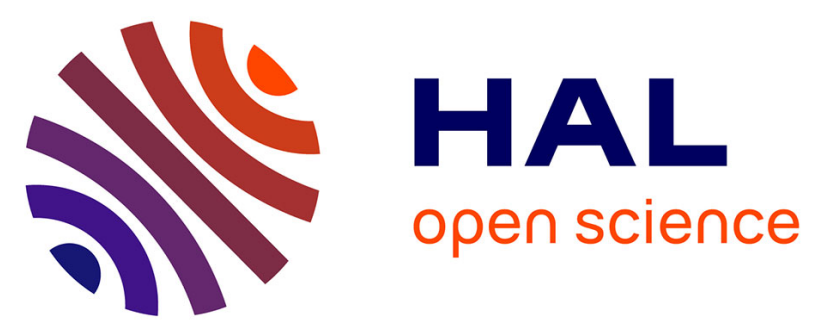

\title{
Tendance à la lapidification de sols sableux (hardé du Nord-Cameroun) Une évolution naturelle sous climat semi-aride à fort pouvoir évaporant
}

\author{
Mathieu Lamotte, Ary Bruand, Georges Pédro
}

\section{- To cite this version:}

Mathieu Lamotte, Ary Bruand, Georges Pédro. Tendance à la lapidification de sols sableux (hardé du Nord-Cameroun) Une évolution naturelle sous climat semi-aride à fort pouvoir évaporant. Comptes rendus de l'Académie des sciences. Série IIa, Sciences de la terre et des planètes, 1997, 325, pp.577-584. 10.1016/S1251-8050(97)89458-X . insu-00860970

\section{HAL Id: insu-00860970}

\section{https://hal-insu.archives-ouvertes.fr/insu-00860970}

Submitted on 12 Sep 2013

HAL is a multi-disciplinary open access archive for the deposit and dissemination of scientific research documents, whether they are published or not. The documents may come from teaching and research institutions in France or abroad, or from public or private research centers.
L'archive ouverte pluridisciplinaire HAL, est destinée au dépôt et à la diffusion de documents scientifiques de niveau recherche, publiés ou non, émanant des établissements d'enseignement et de recherche français ou étrangers, des laboratoires publics ou privés. 


\title{
Tendance à la lapidification de sols sableux (hardé du Nord-Cameroun) Une évolution naturelle sous climat semi-aride à fort pouvoir évaporant
}

\section{Towards the hardening of sandy soils: a natural evolution in semi-arid tropics}

\author{
Mathieu LAMOTTE $^{1 *}$, Ary BRUAND ${ }^{2}$ et Georges PÉDRO ${ }^{3}$ \\ ${ }^{1}$ Département TOALR 12, USP, Instituto de Geociências, ORSTOM, DGG, CP 11.348, 05 422-970 São Paulo-SP, Brésil ; \\ 2 Unité de science du Sol-SESCPF, INRA. Centre d Orleans, 45160 Ardon: \\ ${ }^{3}$ Unité de science du sol, INRA, Centre de Versailles, 78026 Versailles cedex, France
}

\section{RÉSUMÉ}

En région semi-aride, la désertification succède fréquemment à une dégradation des propriétés physiques des sols. Pour les sols sableux, milieux meubles et filtrants, la dégradation qui consiste en l'apparition d'une forte cohésion et d'une faible perméabilité est due au mode d'assemblage des constituants : une faible quantité dargile fine revêt et relie systématiquement les grains du squelette. Ce mode d'assemblage résulte d'une évolution naturelle en relation étroite avec le fonctionnement hydrique, lui-même favorisé par des conditions lithologiques et climatiques. Sans que l'homme n'intervienne nécessairement, des formations supergènes peuvent ainsi perdre les caractèristiques qui en faisaient des sols, à savoir des matériaux meubles, hydratés et biotiques.

Mots clés: Sol induré, Aridification, Sédiment, Nappe, Évaporation, Microorganisation

\begin{abstract}
In semi-arid tropics, the desertification results frequently from the degradation of the physical properties of the soils. For sandy soils, usually friable and permeable, this degradation, which consists of the development of hardness and low permeability, was related to the groundmass fabric. Even at low content, the fine clay formed wall-shaped bridges between the skeleton grains and caused a bigh continuity of tho solid phase. This fabric resulted from natural evolution closely related with water dynamics and favoured by litbology and climate. Without necesarily buman intervention, the supergeneous materials lost their characteristics of heing loose, hydrated and biotic.
\end{abstract}

Keywords: Indurated soil, Aridification, Sediment, Groundwater, Evaporation, Fabric

Abridged version (see p. 583)

Tote présentée par Georges Pédro

Note remise le 9 juin 1997, acceptée le 21 juillet 1997

- E-mail : mlamote@usp.br 


\section{Introduction}

Dans certaines régions du Globe, l'aridité domine en raison du très fort pouvoir évaporatoire de l'atmosphère, et ce malgré une pluviosité annuelle relativement élevée. La désertification s'y manifeste par une diminution progressive, qualitative et quantitative de la productivité biologique des terres (Dregne, 1977 ; Sabadell, 1982 ; Gorse, 1985). Dans la plaine méridionale du bassin tchadien, notamment, de grandes étendues présentent des sols à forte cohésion et faible perméabilité. Elles sont dénudées ou à végétation clairsemée et sont abandonnées par l'homme. Dans le Nord du Cameroun, de telles étendues (hardé) sont généralement associées à des sols peu argileux (Brabant et Gavaud, 1985). Les contraintes édaphiques, mécaniques et hydriques des sols restreignent l'activité biologique à des espèces spécifiques.

L'objectif de cette note, qui s'appuie sur l'étude détaillée d'un site, est de discuter l'origine de la forte cohésion et du pédoclimat aride. On montrera qu'il s'agit très vraisemblablement d'une évolution naturelle, éventuellement accélérée par l'homme, conduisant dans les cas extrêmes à une sorte de "lapidification", du fait de la disparition, difficilement réversible, des caractéristiques définissant un sol - matériau supergène meuble, hydraté et biotique.

\section{Présentation du site}

Le site de Lagadgé $\left(14^{\circ} 43^{\prime} \mathrm{E}\right.$ et $10^{\circ} 25^{\prime} \mathrm{N}$ ) est localisé non loin de Maroua, dans le Nord du Cameroun (figure 1). La région a connu depuis 50000 ans une succession de climats arides et humides, associés respectivement à des dépôts à dominante éolienne et alluviale (Servant, 1973 ; Maley, 1981 ; Durand et Lang, 1986). Le climat actuel est soudano-sahélien. En raison de l'influence saharienne, l'aridité prédomine tout au long de l'année. Flle se manifeste par une forte évapotranspiration (moyenne de $1980 \mathrm{~mm} / \mathrm{an}$ ), une température élevée (moyenne de $28^{\circ} \mathrm{C}$ ) associée à un fort écart diurne, une longue insolation quotidienne (moyenne de $8 \mathrm{~h}$ ) et des vents secs chargés d'aérosols. Les précipitations $(812 \mathrm{~mm} / \mathrm{an})$ se produisent exclusivement pendant 4 à 5 mois. Le site est encadré au sud par un pédiment d'altération du socle, à l'est et à l'ouest par des complexes dunaires et au nord par des alluvions argileuses (Sieffermann, 1963 ; Brabant et Gavaud, 1985). Les sols sont développés au sein de sédiments composites et peu épais recouvrant le socle. Le modelé est peu disséqué et la surface topographique présente une pente de $1 \%$ vers le nord-est. Dans les talwegs, des écoulements temporaires, étroitement associés aux précipitations se produisent du sud-ouest vers le nord-est sur la pédiplaine d'altération du socle. Vers l'aval, en amont du site, les écoulements superficiels sont interrompus (pertes), relayés par des sous-écoulements qui empruntent d'anciens chenaux actuellement enterrés, et alimentent ainsi des nappes temporaires. Le hardé Lagadgé se présente comme une steppe à Balanites agyptiaca,
Lannea humilis et Asparagus flagellaris (zones nues ou zones herbacées de plusieurs hectares), avec des îlots boisés à Guiera senegalensis et Hexalobus monopetalus (zones arborées de quelques ares). La distribution du couvert végétal ne coïncide avec aucune variation systématique de la topographie, et seules de faibles variations de modelé sont associées à des mares, des termitières ou des limites d'érosion (Lamotte, 1995).

\section{Méthodes d'étude}

La couverture de sol a fait l'objet d'une cartographie de résistivité ef d'observations morphologiques dans des fosses le long de séquences (Lamotte et al., 1994). Le fonctionnement hydrique a été suivi à la surface du sol durant la saison humide et des mesures de teneur en eau ont été effectuées dans des fosses, à différentes périodes de l'année (Lamotte, 1995). Le mode d'assemblage des constituants élémentaires a été observé en microscopie sur lames minces. La granulométrie a été étudiée en combinant des techniques de tamisage et de suivi de sédimentation. La composition chimique des constituants fins a été déterminée sur des lames-minces à l'aide d'une microsonde et son interprétation minéralogique s'est appuyée sur des analyses par diffraction des rayons $X$ (Lamotte et al., $1997 \mathrm{a}$ et b).

\section{Résultats et discussion}

\section{Organisation de la couverture de sol et fonctionnement hydrique}

Le matériau parental du sol est constitué d'un substratum alluvial hétérogène (argile, sable fin, sable grossier) et d'un sédiment sablo-limoneux (SL), sus-jacent et homogène, qui résulte de dépôts ou de remaniements éoliens. La discontinuité entre les deux sédiments est soulignée localement par l'interstratification de chenaux de sable grossier (figure 1). Un horizon sableux très cohérent (STC), développé essentiellement au sein du sédiment SL, est généralement présent à faible profondeur, avec une épaisseur de 1 à $2 \mathrm{~m}$ (figure 1). Cet horizon apparaît à la base d'un horizon sableux peu cohérent (SPC), épais de quelques centimètres dans les zones nues, de 25 à $75 \mathrm{~cm}$ dans les zones herbacées, et de 1 à 3 m dans les zones arborées. L'horizon STC est continu, depuis les zones nues jusqu'à proximité des zones arborćes. À l'aplomb de ces dernières, il est généralement absent. La présence de l'horizon STC est associée à une granulométrie argileuse du substratum, tandis que son absence coüncide avec les chenaux de sable grossier (figure 1).

L'humectation saisonnière du sol concerne essentielle. ment I'horizon SPC. Sa teneur en eau est voisine de $1 \%$ en saison sèche et de $15 \%$ en saison humide. L'horizon SPC se dessèche entre les averses, ce qui explique que le maximum de teneur en eau demeure relativement faible, même en saison humide. L'horizon STC est généralement 
Figure 1. a. Localisation du site (hardé Lagadgé). b. Carte de distribution de la végétation et organisation de la couverture de sols. 1: Sédiment supérieur sablolimoneux (SL) ; 2 : substratum (A : argile, $B$ : sable fin, $C$ : sable grossier) ; 3 : nodules ferrugineux ; 4 : horizon sableux peu cohérent $(\mathrm{SPC}) ; 5$ : horizon sableux très cohérent (STC); 6 : éclats de quartzites taillés.

a. Location of the site (Lagadge harde). b. Map of the vegetation pattern and soil cover organisation. 1: Homogeneous upper sandy-loam sediment (SL); 2 : Heterogeneous lower sediment (A: clay, $B$ : fine sand, $C$ : coarse sand); 3: Fe-oxide nodules; 4: sandy horizons with low cohesion (SPC); 5: sandy horizons with high cohesion (STC); 6: lithic quartzite artefacts.

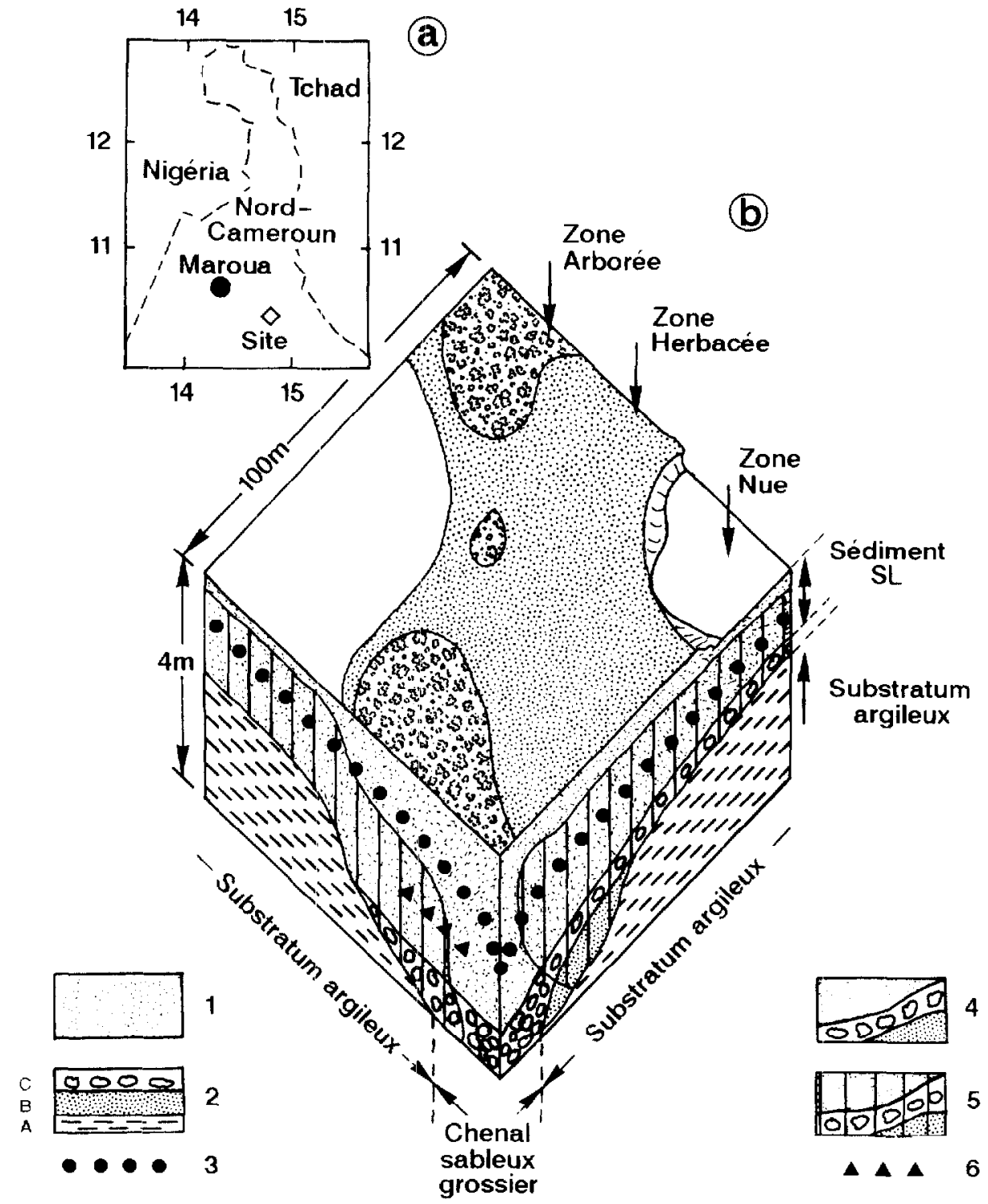

sec. Il ne s'humecte en effet que dans ses premiers centimètres, où la teneur en eau varie de $2 \%$ (saison sèche) à environ $10 \%$ (saison humide). Plus en profondeur, la teneur en eau est toujours faible (environ $3 \%$ ). Dans le sol des zones arborées, I'horizon SPC épais de plusieurs mètres présente une teneur en eau de 3 à $8 \%$ en saison sèche, de 12 à $20 \%$ en saison humide; en outre, une nappe temporaire à $1,8 \mathrm{~m}$ de profondeur a été observée durant quelques semaines en début de saison sèche. Aucun écoulement latéral n'ayant été décelé au contact entre les horizons SPC et STC, l'alimentation de la nappe résulte essentiellement d'un inféro-flux provenant de l'amont du paysage et circulant dans les chenaux de sable grossier, et secondairement d'une infiltration de la lame d'eau précipitée au droit de la zone arborée. L'alimentation de cette nappe par inféro-flux explique d'ailleurs le décalage dans le temps entre la fin des pluies et l'existence de cette nappe.

\section{Mode d'assemblage des constituants élémentaires et propriétés des horizons}

L.'horizon SPC est friable, quelle que soit sa teneur en eau, tandis que l'horizon STC présente une très forte dureté dans son état sec. Après une immersion dans l'eau, les échantillons STC perdent leur cohésion et se dispersent aisément. La discontinuité de consistance entre l'horizon SPC et l'horizon STC coïncide non seulement avec une différence de structure (particulaire et massive) et de porosité ( 39 à $44 \%$ et 28 à $33 \%$ ), mais aussi avec une différence de propriétés hydrauliques : l'humectage est rapide et la perméabilité élevée dans I'horizon SPC, tandis que l'humectage est lent et la perméabilité très faible dans I'horizon STC.

Les horizons SPC et STC différenciés au sein du sédiment homogène SL ont des squelettes similaires. En effet, dans les deux types d'horizons, les grains du squelette 
(>2 $2 \mathrm{~m}$ ) présentent une distribution de taille semblable, une nature analogue (environ $95 \%$ de quartz, $4 \%$ de feldspaths altérés et un même cortège de minéraux lourds) et un mode d'assemblage des grains particulièrement compact et uniforme (Lamotte et al., 1997a). La teneur en argile $<2 \mu \mathrm{m}$ ne permet pas de distinguer les deux horizons ( 2 à $10 \%$ pour l'horizon SPC et 10 à $19 \%$ pour I'horizon STC). En revanche, lors du passage de I'horizon SPC à I'horizon STC, la teneur en argile fine inférieure à $0,2 \mu \mathrm{m}$ augmente systématiquement.

Les observations microscopiques ont montré que les deux horizons sont caractérisés par l'absence de structure microagrégée ou fragmentaire et par la rareté de la porosité d'origine biologique. Dans les deux horizons, les concentrations argileuses (à l'intérieur de cavités ou en coiffe sur des grains) et ferrugineuses (nodules) sont très rares. Dans I'horizon SPC, les grains ne sont généralement ni revêtus, ni reliés entre eux (assemblage de grains nus et libres) ; la fraction argileuse, présente en faible quantité, se répartit en revêtements discontinus sur des grains et à l'intérieur de grains altérés. En revanche, dans l'horizon STC, des cloisons argileuses relient systématiquement les grains et sont en continuité avec des revêtements argileux disposés à leur surface (assemblage cloisonné de grains revêtus). Dans les zones de transition entre les horizons SPC et STC, le nombre de grains revêtus et pontés augmente progressivement, sans discontinuité dans le mode $\mathrm{d}^{\prime}$ assemblage des grains eux-mêmes. Les cloisons sont de forme convexo-concave et leur épaisseur varie avec leur longueur (Lamotte et al., 1997a) : 0,5-1 $\mu \mathrm{m}$ pour les plus longues (10-40 $\mu \mathrm{m})$ et $15-30 \mu \mathrm{m}$ pour les plus courtes (5-10 $\mu \mathrm{m})$. Les déterminations géochimiques et minéralogiques ont montré que les cloisons et les revêtements, de nature homogène, sont composés essentiellement de beidellite alumino-ferrifère et secondairement de kaolinite, d'illite et de quartz (Lamotte et al., 1997b).

L'assemblage cloisonné de grains revêtus est spécifique de l'horizon à forte cohésion. Une teneur en argile de seulement $7 \%$, mais localisée précisément de manière à créer des points de contact entre les grains du squelette, suffit pour établir une continuité élevée de la phase solide. Le liant argileux est à lui seul responsable de la dureté de l'assemblage à sec, car les particules de beidellite alumino ferrifère développent dans cet état une très forte cohésion interparticulaire. En revanche, à l'état humide, après une immersion, ces particules argileuses s'hydratent et la cohésion disparaît. L'absence de porosité structurale ou biologique renforce le caractère de continuité de la phase solide, qui explique non seulement la très forte cohésion, mais aussi la faible perméabilité de l'horizon, et consécutivement l'aridité du pédoclimat.

\section{Activité biologique au sein de la couverture de sol}

Le sol qui présente un horizon SPC épais de plusieurs mètres, avec une humectation saisonnière et une nappe temporaire, est associé à une activité biologique importante, diversifiée et typique des zones arborées (Lamotte, 1995). À l'opposé, en présence d'un horizon STC peu profond et épais, l'activité biologique est réduite et spécifique. L'activité de la mésofaune se résume alors quasi exclusivement à la présence de nids de termites (Odontotermes magdalenæ) qui creusent des galeries et aménagent des cavités (meules à champignons) dans l'horizon STC et qui construisent des cheminées à tra'vers I'horizon SPC jusqu'à la surface du sol. La partie épigée des nids est constituée de cheminées, entourées d'un glacis totalement dépourvu de végétation. Ainsi, Odontotermes magdalenæe aménage un habitacle au sein de l'horizon très cohérent, en se protégeant de toute infiltration d'eau qui pourrait provenir de la surface du sol.

Par ailleurs, l'étude de profils racinaires des deux espèces ligneuses les plus caractéristiques (Balanites ægyptiaca et Lannea humilis) a montré qu'elles développent un pivot vertical au sein de l'horizon SPC et des racines latérales au contact de I'horizon STC. La pénétration racinaire se produit dans I'horizon SPC, en exploitant au maximum l'interface avec l'horizon STC (développement racinaire en baïonnette dans le cas de Balanites ægyptiaca ou avec des organes de réserve hydrique pour Lannea humilis).

Ces observations confirment donc que le développement de l'activité biologique coïncide remarquablement avec l'organisation des horizons (lamotte et al., 1994). La continuité, comme l'extension de I'horizon à très forte cohésion et à pédoclimat aride, expliquent la prédominance des zones désertiques, où ne persiste qu'une activité biologique réduite et spécifique.

\section{Durcissement en relation avec la lithologie, le climat et l'hydrodynamique. Différents stades dans le développement du processus}

Les observations dans des fosses ont montré que la différenciation des horizons SPC et STC est postérieure à la mise en place des sédiments. En effet, la limite entre ces deux horizons est nettement discordante par rapport aux limites sédimentaires (figure 1) : I'horizon STC est développé principalement dans le sédiment supérieur $\mathrm{SL}$, mais aussi dans le substratum (notamment au sein des chenaux de sable grossier). La limite entre les horizons SPC et STC ne peut en aucun cas résulter d'un processus de sédimentation ou d'érosion, les horizons SPC et STC présentant, au sein du matériau supérieur SL, un même squelette (nature, granulométrie et mode d'assemblage similaires). De plus, leur limite est discordante, et donc postérieure, à une concentration d'éclats de quartzites (figure 1) taillés par I'homme (atelier de débitage in situ attesté par la présence d'esquilles de taille associées aux éclats), et découvert à l'occasion d'une prospection géophysique (Lamotte et al., 1994).

Selon toute vraisemblance, la sédimentation (figure 2a) a conduit initialement à la mise en place du substratum à dominante argileuse (régime de décantation). Des intercalations plus grossières se sont mises en place localement, en relation avec un fonctionnement fluviatile (chenaux de sable grossier ou fin). À la faveur de dépôts ou de remaniements éoliens, un sédiment homogène $S L$ a recouvert 
Figure 2. Différenciation de la couverlure de sols en relation avec l'organisation des sédiments, I'hydrodynamique et l'évolution climatique. 1 : Sédiment sablo-limoneux (SL) ; 2 : substratum (A : argile, B : sable fin, $C$ : sable grossier) ; 3 : nodules ferrugineux ; 4 : horizons sableux peu cohérents (SPC) ; 5 : horizons très cohérents (STC); 6 : éclats de quartzites taillés ; 7 : ascension capillaire; 8 : évaporation; 9 : nappe. a. Mise en place du substratum à dominante argileuse (régime de décantation) et des chenaux de sable fin ou grossier (régime fluviatile). b. et c. Dépôt éalien du sédiment sablolimoneux (SL) avec intercalation d'une occupation anthropique dont témoigne un atelier de taille de quartzite. d. Première évolution en climat humide. Existence d'une nappe fortement alimentée par infiltration et/ou inféro-flux dans les chenaux, formation de nodules ferrugineux dans la zone de battement de la nappe. e. Seconde évolution en climat aride. Existence d'une nappe rechargée temporairement par infiltration et/ou inféro-flux dans les chenaux. Dans la zone où se relaient les remontées capillaires et l'évaporation à partir de la surface du sol, l'évaporation et la concentration de la solution du sol (fortement minéralisée) conduisent à la formation de cloisons argileuses (ménisques) dans la porosité intergrains, puis à l'acquisition d'une forte cohésion et à une imperméabilisation, donc au développement de l'horizon sableux très cohérent (STC). Au droit des chenaux, en l'absence de relais entre les remontées capillaires et l'évaporation, la solution du sol ne se concentre pas et le cloisonnement argileux ne se produit pas. f. État actuel. Le functionnement hydrique et l'activité biologique sont très influencés par la présence ou l'absence de l'horizon à forte cohésion et faible perméabilité.

Soil cover differentiation related to sediment organisation, waterflow and climate. 1: Upper sandy-loam sediment (SL); 2: lower heterogeneous sediment ( $A$ : clay, $B$ : fine sand, $C$ : coarse sand); 3: Fe-oxide nodules; 4: sandy horizons with low cohesion (SPC); 5: sandy horizons with high cohesion (SIC); 6: capillary rise; 7: lithic quartzite artefacts; 8: evaporation: 9: groundwater table.

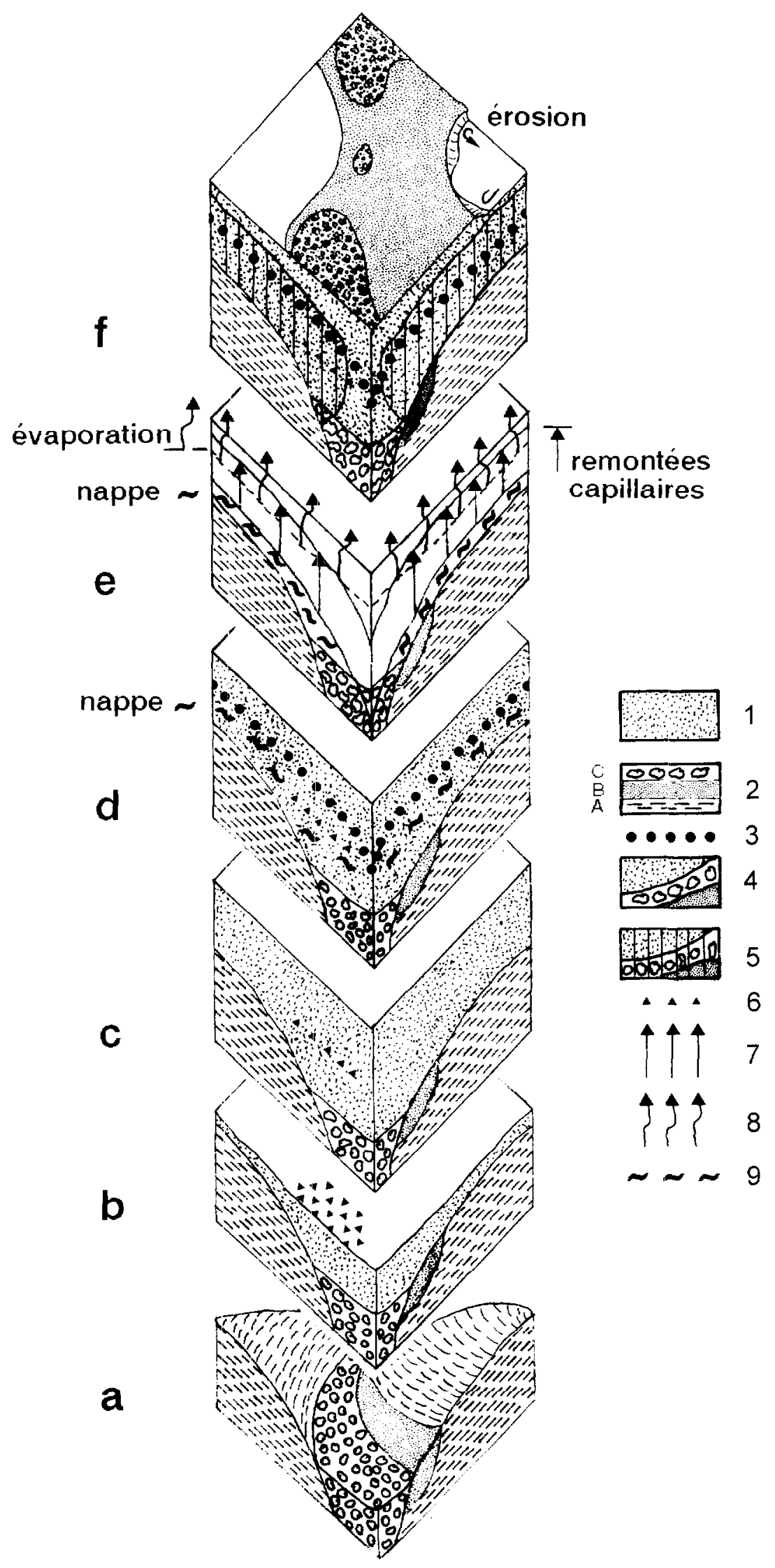


le substratum. L'atelier de débitage de quartzite témoigne d'une occupation humaine entre deux phases de dépôt du sédiment SL (figure $2 \mathrm{~b}$ et $\mathrm{c}$ ). Une telle succession de dépôts est conforme à l'existence de variations paléoclimatiques entre des périodes humides (dépôts fluviolacustres) et des périodes arides (dépôts ou remaniements éoliens).

Par ailleurs, la présence de l'horizon SIC est associée à une granulométrie argileuse du substratum, alors que, a contrario, son absence coïncide avec une granulométrie sableuse (figure 1). Ainsi, le caractère imperméable ou perméable du substratum a déterminé le développement ou l'absence de l'horizon STC. La dynamique de l'eau et plus particulièrement l'existence temporaire d'une nappe expliqueraient la formation de l'horizon STC. Cette formation a été favorisée par la superposition d'un sédiment initialement perméable (SL) sur un substratum argileux imperméable qui a joué le rôle de plancher hydraulique pour une nappe.

Lors d'une première phase de pédogenèse liée à un paléoclimat humide (figure $2 \mathrm{~d}$ ), une nappe se développe à la base du sédiment SL. Cette nappe a pu être alimentée par une infiltration des pluies à la surface du sol et par un inféro-flux dans les chenaux de sable grossier. La formation de nodules ferrugineux, alignés selon des niveaux de battement de la nappe (figure 1), daterait de cette phase de pédogenèse, qui est également associée à un fonctionnement éluvial-illuvial dont subsistent des indices, rares, mais caractéristiques (concentrations argileuses à l'intérieur de cavités ou en coiffe sur des grains).

Une seconde phase d'évolution, dans les conditions du climat actuel, contrasté et plus aride, est responsable de la formation de l'horizon STC. A la base du sédiment SL, la nappe n'est présente que temporairement lors de périodes humides. Compte tenu de la taille des pores (Lamotte et al., 1997a), des remontées capillaires peuvent se produire sur une hauteur de $1,5 \mathrm{~m}$ à partir du toit de la nappe (figure $2 \mathrm{e}$ ). Au cours des périodes sèches, l'évaporation de la solution du sol aurait entraîné la néoformation et/ou la concentration de minéraux argileux préalablement présents en suspension, ceux-ci se déposant sur la surface des grains et dans les espaces intergrains, en créant des points de jonction. La répétition du cycle conduisant à la recharge de la nappe, puis à la concentration de la solution du sol par évaporation, est responsable de la formation de l'assemblage cloisonné de grains revêtus. L'eau ne pouvant plus pénétrer à travers l'assemblage cloisonné, qui se met en place après chaque phase de dessiccation, le matériau sablo-limoneux ayant acquis un tel assemblage élémentaire demeurerait de plus en plus sec et acquerrait de la sorte une très forte cohésion. Seul ce scénario explique le développement uniforme et continu de l'horizon STC, sur de grandes distances et sur une grande épaisseur.
I.ocalement, en présence de chenaux de sable grossier, le toit de la nappe élant situé à une plus grande profondeur, les remontées capillaires n'ont pas atteint le front d'évaporation et la concentration de la solution du sol n'a pas pu se produire (figure $2 f$ ). De plus, l'inféro-flux dans les chenaux de sable grossier a pu jouer un rôle de soutirage, réduisant ainsi le phénomène de concentration de la solution du sol. En présence de tels chenaux, le fonctionnement hydrique n'aurait donc pas été favorable à la formation de l'horizon STC, expliqua'nt par conséquent son faible développement, ou même son absence.

\section{Conclusion}

Selon le schéma d'évolution proposé, la différenciation des horizons SPC et STC résulte d'une évolution naturelle, en relation avec des conditions particulières d'organisation des sédiments, de climat et de fonctionnement hydrique. Bien qu'aucune véritable cimentation n'ait été observée, l'évolution décrite conduit bien à une sorte de "lapidification", dans le sens où des matériaux après avoir été initialement meubles, hydratés et biotiques, ne le sont plus. Le développement de grandes êtendues hardé présentant des sols à forte cohésion et faible perméabilité apparaît comme la conséquence d'une évolution régressive de la pédogenèse, dans les régions où le climat semi-aride est relativement pluvieux, mais à fort pouvoir évaporanl.

Le fonctionnement hydrique actuel est très influencé par la présence de l'horizon à très forte cohésion et faible perméabilité : l'aridité est permanente darıs le sol dominant à horizon STC peu profond et l'humectation n'est que saisonnière dans le sol à horizon SPC épais, où se développe localement une nappe temporaire. La diminution, difficilement réversible, de la biodiversité qui accompagne cette évolution, se fait au profit d'espèces spécifiques qui maintiennent une faible activité biologique, remarquablement adaptée à la succession de courtes périodes pluvieuses, puis de longues périodes très arides. L'activité humaine n'intervient pas nécessairement dans le scénario d'évolution conduisant à la formation de l'horizon sableux très cohérent. Néanmoins, l'intervention de l'homme peut encore réduire, voire faire disparaître l'activité biologique dans de tels sols, accélérant ainsi l'évolution vers leur "lapidification".

Ainsi, notre étude montre que, non seulement des formations supergènes peuvent perdurer sans évolution sous climat semi-aride (déserts minéraux), mais qu'elles peuvent aussi, après avoir connu une évolution ayant conduit à la différenciation de sols, connaître une évolution pédologique régressive, conduisant à leur durcissement et leur imperméabilisation. 


\section{$\triangle$ BRIDGED VERSION}

The desertification of the soils under sudann-sahelian climate. (Dregne, 1977; Sabadell, 1982; Gorse. 1985) is closely related to the degradation of their physical properties (hardness and low permeability). This degradation occurred in large areas of sandy soils (Brabant and Gavaud, 1985) which, consequently, support specific and reduced biological activities. A study was carried out on the site of Lagadge harde (Lamotte, 1995) located in northern Cameroon (figure 1). The mean annual precipitation (May-September) and the mean annual potential cvapotranspiration are 812 and $1980 \mathrm{~mm}$ respectively. The outflow which occurred temporarily in the thalwegs during the wet season is interrupted upstream from the site, and relayed by underflow towards buried channels (Sieffermann, 1963). On the site (figure 1), the topography was flat and large bare or herbaceous areas alternated with small arboreous areas.

The parent material (figure 1) consisted of (i) a heterogeneous alluvial substratum (predominantly clayey with sandy channels) and (ii) an overlying homogeneous aeolian sandy-loam sediment (SL). Two horizons developed mainly in the SL sediment: a loose horizon (SPC) variable in thickness overlying a hard horizon (STC). The SPC horizon is only a tew centimetres thick in the bare areas, a few decimetres thick in the herbaceous areas, and is absent in the areas wilh trees. The presence of the STC horizon close to the surface is associated with the presence of a clayey substratum while its absence coincicies with the sandy channels. The seasonal rewetting of the snil concerned mainly the SPC horizon since the rainwater infiltrated concerned the first upper centimetres of the SIC horizon. The water content is permanently very low at the bottom of the STC horizon. In the soil with an SPC horizon $3 \mathrm{~m}$ thick, a groundwater table occurred late in the wet season and early in the dry season. Without lateral waterflow in the SPC horizon, the recharge of the groundwater resulted primarily from lateral throughflow towards the sandy buried channels (lateral waterflow originating from upstream of the landscape) and secondarily from vertical percolation of the rainwater through the soil.

The SPC horizon is always friable, whereas the STC horizon is extremely firm in the more currently dry state and it slakes when immersed in water. On the profiles, the variation in consistence from the SPC horizon to the STC horizon corresponded to a difference in structure (from a single grain to a massive structure) and porosity (from $39-44 \%$ to $28-33 \%$ ). The SPC and STC horizons which derived from the sandy-loam sediment (Lamotte et al., 1997a) had similar skeleton grains, without any difference in the distribution of particles $>2 \mu \mathrm{m}$, the mineralogical composition and the packing of the grains (particularly uniform and dense). The SPC and STC horizons cannot be distinguished on the basis of the total clay content, but the STC horizon differs from the SPC horizon by a small increase in the fine clay particles $<0.2 \mu \mathrm{m}$. The STC horizon was characterized by a specific fabric with clay coatings on the skeleton grains and clay wall-shaped bridges between the latter. The coatings and the bridges consist primarily of $\mathrm{Al}-\mathrm{Fe}$ beidellite, with small contents of kaolinite, illite and quart?. (Lamotte et al., 1997b). In the dry state, the cohesion between the beidellite particles ensures continuity of the solid phase and the hardness of the soil. When immersed in water, the interparticle cohesion decreases and the soil disperses easily. The absence of a crack network explains the considerable strength of the STC horizon and the difficulties for water infiltration.

Because of the water availability, the soil with $3 \mathrm{~m}$ thick SPC horizon showed dense and diversified flora, and numerous species of mesofauna (termite, earthworm, ant. In contrast, in the other parts of the soil cover, the STC horizon close to the soil surface restricted biological activity. The two specific species of trees (Balanites cegypliaca and Jamnea bumilis) exhibited horizontal roots at the bottom of the SPC horizon which did not penetrate the STC horizon. The Odontermes magdalence termitarias (specific mesofaunal acivity) consisted of a habitat of vugs, excavated within the STC horizon, and interconnected chimneys which were built by the termites at the soil surface. The chimneys play the role of ventilating shaft and impediment to the infiltration of rainwater.

According to the variation of climate cluring the Quaternary (Servant, 1973; Maley, 1981; Durand and Lang, 1986), alluvial and aeolian processes of sedimentation were successively responsible for the deposits of the heterogeneous substratum and of the upper homogeneous SL sediment ifigure 2a). The archaeological layer discovered in 1990 during, a geoelectrical survey (Lamotte et al., 1994) and composed of lithic (quartzite) artefacts from in situ debitage indicatcd a human occupation of the site between two phases of aeolian sedimentation (figures $2 \mathrm{~b}$ and $\mathrm{c}$ ). The STC horizon intersected the limit between the two sediments and the archaeological layer, thus indicating that the STC horizon differentiated later on the phases of sedimentation (figure 1). During a wet palaeoclimate, a groundwater table occurred in the SL sediment (figure $2 \mathrm{~d}$ ). Its recharge was related both to vertical infiltration of rainwater and lateral underflow towards the intercalated sandy channels. Alignments of Fe-oxide nodules resulted from perched watertable fluctuations (figure 1). The formation of the STC horizon was favoured by the current climate which is more arid and contrasted than before. The groundwater occurred temporarily during wet periods in the lower part of the SL sediment. The discharge of the groundwater during dry periods was likely the result of capillary rise upwards from the watertable and evapotranspiration from the soil surface (figures $2 \mathrm{e}$ and $\mathrm{f}$ ). The formation of the wall-shaped fabric which appeared as specific of the STC horizon could be caused by the evaporation of the soil solution containing fine clay in suspension (minerals inherited or formed in situ). The succession of groundwater recharges and soil solution concentrations by evaporation would be responsible for the genesis of the wall-shaped fabric, the decrease of the porosity, and, consequently, the decrease of the permeability. Iorally, where the sandy channels were buried under the SL sediment, the absence of the clayey substratum was associated with the deepening of the groundwater table and its capillary rise (figure 2e). Therefore, the conditions were unfavourable to the concentration of the soil solution by evaporation and corisequently to the genesis of the wall-shaped fabric. 
In conclusion, the formation of an STC horizon is favoured by the low thickness of a permeable sediment overlying an impermeable substratum. During wet periods, a groundwater capillary rise occurred in the permeable sediment. The concentration of the soil solution during periods of high evaporation was responsible for the clay bridging which ensures the continuity of the solid phase and explains the strong cohesion and the low permeability of the horizon. Thus, the development of the hardness and aridity of the pedoclimate is closely related with the characteristics of the sediments, the water dynamics and the climate. Human activities did not directly play a role in this development, but could favor the disappearance of specific biological activities which are adapted to the arid pedoclimate.

Remerciements : Nous remercions M. Gavaud e† F.X. Humbel (ORSTOM) pour leur contribution à notre rëflexion, S. Ledermann (Roussel-Uclaf) pour son aide lors de l'étude floristique et $S$. Desbourdes (INRA) pour son aide lors de la réalisation des illustrations.

\section{RÉFÉRENCES}

Brabant P. et Gavaud M. 1985, Les sols et les ressources en terres du Nord-Cameroun. Notice explicative. ORSTOM. Paris, 103, $285 \mathrm{p}$.

Dregne H.E. 1977. Status of desertification in the hot arid regions, climate aridity index map, experimental world scheme of aridity and drought probability at a scale of $1 / 25000000$. Explanatory note, UNEP, Nairobi, $12 \mathrm{p}$.

Durand A. et Lang J. 1986. Approche critique des méthodes de reconstitution climatique: le Sahel nigéro-tchadien depuis 40000 ans, Bull. Soc. géol. France, 8, (II, 2), 267-278

Gorse J. 1985, La désertification dans les zones sahéliennes et soudaniennes de l'Afrique de l'Ouest. Bull. Rech. Agron. Gembloux, 20, (3-4), 943-1104

Lamotte M. 1995. Les sols sableux à forte cohésion des zones tropicales arides. Étude du hardé Lagadgé au Nord-Cameroun, Thèse, Univ. Paris VI, TDM, ORSTOM, Paris, $134,315 \mathrm{p}$

Lamotte M., Bruand A., Dabas M., Donfack P., Gabal da G., Hesse A. Humbel F.X. et Robain H. 1994. Distribution d'un horizon à forte cohésian au sein d'une couverture aride du Nord-Cameroun: apport d'une prospection électrique, C. R. Acad. Sci. Paris, 318 , série $11.961-968$

Lamotte M., Bruand A., Humbel F. X., Herbillon A. et Rieu M. 1997a A hard sandy-loam soil from semi-arid Northern-Cameroon. I. Fabric of the groundmass, Eur. J. Soil Sc., 48, 213-225

Lamotte M. Bruand A., Ohnenstetter D., lldefonse P. et Pédro G. 1997b. A hard sandy-loam soll from semi-arid NorthernCameroon. II. Geochemistry and mineralogy of the bonding ugent. Eur. J. Soil Sci., 48, 227-237

Maley J. 1981. Études palynologiques dans le bassin du Tchad ef paléoc imatologie de l'Afrique nord-tropicale de 30000 ans à l'époque actuelle, Trav. et DOC. ORSTOM, Paris, 129,586 p

Sabadell J.E. 1982. Systematic approach to desertification. G. Washington University, Washington DC.

Servant M. 1973. Séquences continentales et variations climatiques: évolution du bassin du Tchad au Cénozolique supérieur, Thèse Sc., Univ, Paris VI, multigraphié, $348 \mathrm{p}$.

Sieffermann G. 1963. Carte pédologique du Nord-Cameroun à $1 / 100000$. Feulle de Kalfou Notice de carte, ORSTOM, Yaoundé. $65 \mathrm{p}$. 